\title{
SEMI-INFINITE WAVEGUIDE \\ MADE OF PIEZOELASTIC MATERIAL OF CLASS 6MM
}

\author{
M. V. Belubekyan, A. A. Papyan, \\ Institute of Mechanics National Academia Since Armenia, Yerevan, Armenia, \\ mbelubekyan@yahoo.com,aro088@mail.ru
}

DOI: $10.36724 / 2664-066 X-2020-6-2-16-19$

\begin{abstract}
The problems of coupled physical fields, such as the interaction of mechanical and electromagnetic fields, piezoelectric effect, electrostriction and others, are the most urgent. The study of the issues of wave propagation in piezoelectric materials is also relevant. In this paper the propagation of a monochromatic electroelastic signal in a semi-infinite piezoelectric layer is considered. Let's consider different cases of the boundary conditions, from which localized vibrations can be obtained in the vicinity of the free edge.
\end{abstract}

KEYWORDS: piezoelectric, wave propagation, waveguide, vibration, frequency, Bleustein-Gulyayev waves.

\section{INTRODUCTION}

The problems of wave propagation in piezoelastic media are described in [1-9]. In [1, 8], the appearance of internal resonance in the case of propagation of a monochromatic electroelastic signal in a piezoelectric layer is considered. In [4], a system of a layer and a half-space of elastic and piezoactive materials is considered, the layer and half-space can freely slide relative to each other. The work [5] considers the identification of qualitative effects in the case of propagation of a monochromatic electroelastic signal in piezoceramic, dielectric and inhomogeneous layers over the thickness. This paper considers the propagation of a monochromatic electroelastic signal in a semi-infinite piezoelectric layer. 


\section{Variable separation method}

Various variants of the combination of boundary conditions are considered, some of which allow using the method of separation of variables.

A piezoelectric layer of hexagonal symmetry of class $6 \mathrm{~mm}$ in a rectangular Cartesian coordinate system 0xyz is located so that the $0 \mathrm{z}$ axis is parallel to the symmetry axis of the piezoelectric layer, and the 0xy plane is the plane of symmetry of the piezoelectric. A piezoelectric layer in a rectangular Cartesian coordinate system occupies $0<x<\infty ; 0 \leq y \leq h ;-\infty<z<\infty$. region (Figure 1).

The equations of propagation of a plane purely shear electromechanical wave can be written in the following form [2].

$$
c_{t}^{2} \Delta w=\frac{\partial^{2} w}{\partial t^{2}}, \Delta \psi=0
$$

where

$$
\begin{aligned}
& c_{t}^{2}=\frac{\tilde{C}_{44}}{\rho}, \tilde{C}_{44}=C_{44}(1+\chi), \\
& \chi=\frac{e_{15}^{2}}{\varepsilon C_{44}}, \quad \psi=\varphi-\frac{e_{15}}{\varepsilon} w .
\end{aligned}
$$

$w$ - shear offset, $C_{44}$ - shear modulus, $\rho$ - material density, $\mathcal{E}$ - dielectric constant, $e_{15}$ - piezoelectric module, $\chi$ - electrical connection coefficient, $\varphi$ - electrical potential.

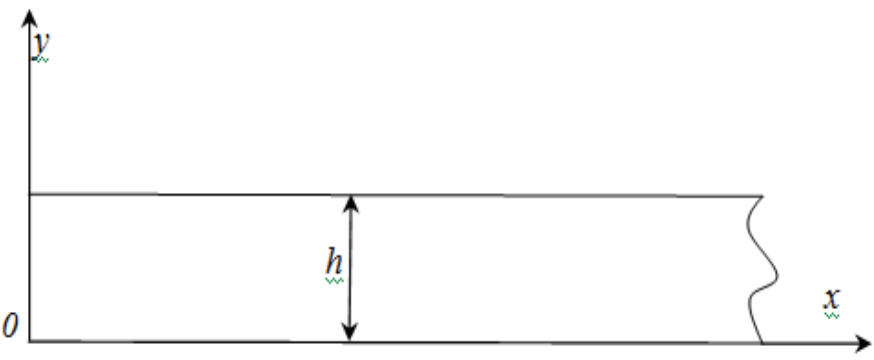

Figure 1. Location of the piezoelectric layer in a rectangular Cartesian coordinate system

For $y=0$ and $y=h$ three variants of boundary conditions are possible, which allow using the method of separation of variables:

$$
\begin{aligned}
& w=0, \quad \varphi=0 \text { or } w=0, \quad \psi=0 \\
& \sigma_{23}=0, \quad D_{2}=0 \text { or } \frac{\partial w}{\partial y}=0, \quad \frac{\partial \psi}{\partial y}=0 \\
& w=0, \quad D_{2}=0 \text { or } w=0, \quad \frac{\partial \psi}{\partial y}=0
\end{aligned}
$$

The second forms of the boundary conditions follow from taking into account the material equations:

$$
\begin{gathered}
\sigma_{31}=C_{44} \frac{\partial w}{\partial x}+e_{15} \frac{\partial \varphi}{\partial x}, \sigma_{32}=C_{44} \frac{\partial w}{\partial y}+e_{15} \frac{\partial \varphi}{\partial y} \\
D_{1}=-\varepsilon \frac{\partial \varphi}{\partial x}+e_{15} \frac{\partial w}{\partial x}, D_{2}=-\varepsilon \frac{\partial \varphi}{\partial y}+e_{15} \frac{\partial w}{\partial y}
\end{gathered}
$$

$\sigma_{31} ; \sigma_{32}-$ stress tensor components, $D_{i}-$ electric induction vector components.

Border conditions $\sigma_{23}=0, \varphi=0$, at $y=0$ and $y=h$ do not separate variables.

Let on planes $y=0$ and $y=h$ the boundary conditions (3) hold. Then the solution to equation (1) satisfying conditions (3) will be represented in the following form:

$$
\begin{aligned}
& w=\sum_{n=1}^{\infty} w_{n}(x) \sin \left(\lambda_{n} y\right) \exp \left(i \omega_{n} t\right), \\
& \psi=\sum_{n=1}^{\infty} \psi_{n}(x) \sin \left(\lambda_{n} y\right) \exp \left(i \omega_{n} t\right), \quad \lambda_{n}=\frac{\pi n}{h}
\end{aligned}
$$

To solve the problem of the possibility of the existence of localized oscillations (waves) in the vicinity of the free edge $x=0$ of a semi-infinite waveguide, it is necessary that the conditions:

$$
\lim _{x \rightarrow \infty} w=0, \quad \lim _{x \rightarrow \infty} \psi=0
$$

Substitution of (2) into equations (7) leads to successive ordinary differential equations with respect to $w_{n}(x), \psi_{n}(x)$ :

$$
w_{n}=A_{n} \exp \left(-\lambda_{n} \sqrt{1-\eta_{n}} x\right), \quad \psi_{n}=B_{n} \exp \left(-\lambda_{n} x\right)
$$

Here

$$
\eta_{n}=\omega_{n}^{2} c_{t}^{-2} \lambda_{n}^{-2}
$$

The dimensionless sought-for characterizing phase velocity must satisfy the condition:

$$
0<\eta_{n}<1
$$

If at the waveguide boundary $x=0$ conditions of type (3) (5) are given, for example:

$$
w=0, \quad \frac{\partial \psi}{\partial x}=0, \quad\left(D_{1}=0\right)
$$


$\mathrm{t}$ is easy to show that a solution satisfying condition (11) does not exist, that is, localized oscillations are not possible.

Let the conditions be given at the edge of a semi-infinite waveguide:

$$
\sigma_{12}=0, \quad \varphi=0 \text { при } x=0
$$

Condition (13) can be reduced to the following form:

$$
C_{44}(1+\chi) \frac{\partial w}{\partial x}+e_{15} \frac{\partial \psi}{\partial x}=0, \psi+\frac{e_{15}}{\varepsilon} w=0
$$

The requirement that solution (9) satisfies boundary conditions (14) leads to a system of algebraic equations for arbitrary constants $A_{n}, B_{n}$. From the condition that the determinant of this system is equal to zero, it is possible to determine the dimensionless parameter of the phase velocity.

$$
\eta=1-\frac{\chi^{2}}{(1+\chi)^{2}}
$$

The dimensionless quantity obtained in equation (15), which characterizes the phase velocity, coincides with the phase velocity for the Bluestein - Gulyaev waves. However, if for Bluestein Gulyaev waves $\eta=\omega^{2} c_{t}^{-2} k^{2}$ is a continuous spectrum of eigenvalues, (for an arbitrary wave number $k$ ), here this spectrum is discrete.

In the case when the boundary conditions of variant (4) are given on the semi-infinite sides of the waveguide, solution (7) will be written:

$$
\begin{aligned}
& w=\sum_{n=1}^{\infty} w_{n}(x) \cos \left(\lambda_{n} y\right) \exp \left(i \omega_{n} t\right), \\
& \psi=\sum_{n=1}^{\infty} \psi_{n}(x) \cos \left(\lambda_{n} y\right) \exp \left(i \omega_{n} t\right), \quad \lambda_{n}=\frac{\pi n}{h}
\end{aligned}
$$

It is easy to show that, similarly to the previous case, satisfying Eq. (1) and boundary conditions (14), we obtain expression (15) for the phase velocity parameter.

The option is assumed when on the planes of the plate $y=0$ and $y=h$ different types of boundary conditions are set:

$$
\begin{aligned}
& w=0, \quad \psi=0, \text { at } y=0 \\
& \frac{\partial w}{\partial y}, \quad \frac{\partial \psi}{\partial x}=0, \quad \text { at } y=h
\end{aligned}
$$

A solution to equation (1) satisfying the boundary conditions (7) can be represented as:

$$
\begin{aligned}
& w=\sum_{n=1}^{\infty} w_{n}(x) \sin \left(\mu_{n} y\right) \exp \left(i \omega_{n} t\right), \\
& \psi=\sum_{n=1}^{\infty} \psi_{n}(x) \sin \left(\mu_{n} y\right) \exp \left(i \omega_{n} t\right), \\
& \mu_{n}=\frac{(2 n-1) \pi}{2 h}
\end{aligned}
$$

Carrying out a procedure similar to the solution of the previous versions, we obtain for the phase velocity parameter the expressions (15) where it is necessary to replace $\lambda_{n}$ by $\mu_{n}$. Hence it follows that the phase velocity of the localized wave does not change, but the frequencies and wavelengths change.

Now let the conditions:

$$
w, \frac{\partial \psi}{\partial y}=0, \quad \text { при } y=0, y=h
$$

The solution to equation (1) in this case will have the following form:

$$
\begin{aligned}
& w=\exp (i \omega t) \sum_{n=1}^{\infty} w_{n}(x) \sin \left(\lambda_{n} y\right), \\
& \psi=\exp (i \omega t) \sum_{n=1}^{\infty} \psi_{n}(x) \cos \left(\lambda_{n} y\right), \\
& \lambda_{n}=\frac{\pi n}{h}
\end{aligned}
$$

Obviously, the solution for functions $w_{n}, \psi_{n}$ will be the same as solution (9).

However, boundary conditions (14), for $x=0$, do not lead to separation of variables.

To satisfy the boundary conditions (14), the averaging method is used, as is done when applying the Bubnov - Galerkin method. Solution (20) is substituted into condition (14), then the first condition is multiplied by $\sin \left(\lambda_{m} y\right)$, second on $\cos \left(\lambda_{m} y\right)$, ( $m=1,2,3 \ldots$ ) and integrating over $y$ in the range from 0 to $h$.

$$
\begin{aligned}
& \sum_{n=1}^{\infty}\left[C_{44}(1+\chi) w_{n}^{1}(0) \int_{0}^{h} \sin \left(\lambda_{m} y\right) \sin \left(\lambda_{n} y\right) d y+e_{15} \psi_{n}^{1}(0) \times\right. \\
& \left.\times \int_{0}^{h} \sin \left(\lambda_{m} y\right) \cos \left(\lambda_{n} y\right) d y=0\right] \\
& \sum_{n=1}^{\infty}\left[\frac{e_{15}}{\varepsilon} w_{n}(0) \int_{0}^{h} \cos \left(\lambda_{m} y\right) \sin \left(\lambda_{n} y\right) d y+\psi_{n}(0) \times\right. \\
& \left.\times \int_{0}^{h} \cos \left(\lambda_{m} y\right) \cos \left(\lambda_{n} y\right) d y=0\right]
\end{aligned}
$$

System (21) $(m=1,2,3 \ldots)$ after calculations is reduced to the form: 


$$
\begin{aligned}
& C_{44}(1+\chi) w_{m}^{1}(0)+\frac{2 e_{15}}{\pi} \sum_{n=1}^{\infty} \psi_{n}^{1}(0) \frac{m\left(1-(-1)^{m+n}\right)}{m^{2}-n^{2}}=0 \\
& \frac{2 e_{15}}{\pi \varepsilon} \sum_{n=1}^{\infty} \frac{n\left(1-(-1)^{m+n}\right)}{n^{2}-m^{2}} w_{n}(0)+\psi_{m}(0)
\end{aligned}
$$

The resulting system (22) in the approximation $(m=1)$ and $(m=2)$ :

$\lambda_{1} \sqrt{1-\eta_{1}} C_{44}(1+\chi) A_{1}-+\frac{4 e_{15}}{3 \pi} \lambda_{2} B_{2}=0$

$\frac{8 e_{15}}{3 \pi \varepsilon} A_{2}+B_{1}=0$

$\lambda_{2} \sqrt{1-\eta_{2}} C_{44}(1+\chi) A_{2}-+\frac{8 e_{15}}{3 \pi} \lambda_{1} B_{1}=0$

$\frac{4 e_{15}}{3 \pi \varepsilon} A_{1}-B_{2}=0$

Hence, for the first and second frequencies, we obtain:

$$
\eta_{1}=1-\frac{32 \chi^{2}}{9 \pi^{2}(1+\chi)^{2}}, \quad \eta_{2}=1-\frac{16 \chi^{2}}{9 \pi^{2}(1+\chi)^{2}}
$$

Comparison of the values of phase velocities (24) and (15) shows that in the case of boundary condition (19) at $x=0$ the velocity of localized waves is higher, which leads to a comparatively slow decay.

\section{Conclusion}

It was found that the method of separation of variables is used for two boundary conditions. It is shown that for these conditions there are localized oscillations, the phase velocity parameter coincides with the Blyustein - Gulyaev formula, but in con- trast to Bluestein - Gulyaev, here the spectrum of eigenvalues is discrete. The phase velocity of the localized wave does not change, but the frequencies and wavelengths do.

When it is impossible to apply the method of separation of variables to satisfy the conditions, the Bubnov - Galerkin method is used. Comparison of the dimensionless values of the phase velocity shows that in this case the velocities of localized waves are higher, which leads to a comparatively slow decay.

\section{References}

1. Belubekyan V.M., Belubekyan M.V. Resonant and localized shear vibrations in a layer with a rectangular cross section. Reports of the NAS of Armenia, 2015. Vol. 115, no. 1. P. 40-43.

2. Bardzokas D.I., Ku yavtsev B.A., Senik N.A. Propagation of waves in electromagnetoelastic media. Editorial URSS, 2003. 336 p.

3. Bleustein J.L. Some simple modes of wave propagation in an infinite piezoelectric plate. Journ. of Acustical Society of America, 1969, 45, pp. 614-620.

4. Belubekyan M.V., Belubekyan V.M. Surface waves in piezoactive elastic system of a layer on a semi-space. Proceedings of the Yerevan State University, Physical and Mathematical Sciences. Mechanics. 2013, no. 3, pp. 45-48.

5. Avetisyan A.S., Kamalyan A.A. Influence of transverse inhomogeneity of a piezodielectric layer and combinations of boundary conditions on the propagation of a shear electroelastic signal. SEUA Bulletin. series "Mechanics, mechanical engineering, mechanical engineering". 2014. Issue 17, no. 1. P. 9-25.

6. Vilde M.V. Kaplunov Yu.D., Kossovich L.Yu. Edge and interface resonances of the phenomenon in elastic bodies. Moscow: Fizmatlit, 2010. 280 p.

7. Gulyaev Yu.V. Surface electrosonic waves in solids. JETP Letters. 1969. Vol.4 no. 1. P. 63-65.

8. Belubekyan M.V., Papyan A.A. On the problem of wave propagation in a layer made of piezoelastic material of $6 \mathrm{~mm}$ class. Actual problems of continuum mechanics: Proceedings of the IV international conference, September 21-26, 2015, Tsaghkadzor, Armenia. P. 91-94. 